\title{
La yerba no es basura: lombricultura y producción de Vermicompost a partir de residuos de yerba mate en Uruguay
}

\author{
Torrendel, M. ${ }^{(1)}$, Useta, G. ${ }^{(2)}$, Pelerino, F. (3) \\ (1) Departamento de Microbiología, Laboratorio Tecnológico del Uruguay (LATU) \\ ${ }^{(2)}$ Departamento de Medio ambiente, Laboratorio Tecnológico del Uruguay (LATU) \\ (3) Centro Uruguay Independiente (CUI) \\ Contacto: mtorrendel@latu.org.uy \\ Recibido: 26/9/2008 - Aprobado: 30/12/2008
}

\begin{abstract}
$\underline{\text { Resumen }}$
En Uruguay el consumo anual de yerba mate genera unas 125 toneladas de residuos orgánicos. La lombricultura es una de las formas de obtener materia orgánica a partir del reciclado de residuos domiciliarios orgánicos. Los desechos de yerba mate tienen un gran potencial de reciclado si son utilizados como sustrato en la lombricultura. Utilizando distintos sustratos, tiempos de compostaje y volúmenes de yerba, se determinaron algunas de las condiciones necesarias para la utilización de dichos residuos como sustrato para la lombriz Eisenia fetida. Se estudiaron las condiciones de pH que el desecho debería alcanzar para ser tolerado y mejor aprovechado por las lombrices, y el período de tiempo que la yerba demora en alcanzar estas condiciones. Se observó que el período de tiempo mínimo de precompostado para el mejor aprovechamiento de la yerba por parte de las lombrices es de cuatro semanas.

Se analizaron también las características del vermicompost obtenido, en cuanto a parámetros de materia orgánica, nitrógeno, fósforo y metales. El valor de nitrógeno (1\%) y de materia orgánica, que ronda el $30 \%$, son similares a los encontrados en otros humus que se obtienen por métodos semejantes. Sin embargo, para el Fósforo los valores resultaron por debajo de lo encontrado en los análisis realizados por el CUI (Laboratorio Ambiental Echotech, octubre 2006).

Palabras clave: vermicompost, lombricultura, yerba mate, reciclaje, residuos orgánicos.
\end{abstract}

\begin{abstract}
$\underline{\text { Abstract }}$
The annual consumption of "yerba mate" (mate herb infusion) in Uruguay generates about 125 metric tons of organic waste. Vermicomposting is one way to obtain organic matter by recycling organic household waste. The waste of "yerba mate" has a great potential for recycling if it is used as a substrate in vermicomposting. Using different substrates, composting times and volumes of "yerba mate" we determined some of the necessary conditions for the use of yerba mate waste as a substrate for the Eisenia fetida earthworm. We studied the optimal pH conditions that the waste should have in order to be tolerated and best exploited by the worms and the period of time that the "yerba mate" takes to achieve these conditions. We observed that four weeks is the minimum amount of precomposting time of "yerba mate", recquired by the worms for an improved processing of it. The vermicompost obtained was analyzed. The following analysis were performed: organic matter, Nitrogen, Phosphorous and metals. The values found for Nitrogen $(1 \%)$ and organic matter (which was around $30 \%$ ) are parallel to the values found for other composts which are obtained by similar composting methods. However, the value of Phosphorus was found to be lower than that found in the analysis performed by CUI (Ambiental Laboratory Echotech, October 2006).

Key words: vermicomposting, worm casting, yerba mate, recycling, organic waste.
\end{abstract}

\section{Introducción}

El consumo de yerba mate, Ilex paraguayensis (Arquifoliaceae), cuyas hojas tostadas y desmenuzadas se beben en infusión, está ampliamente extendido en la sociedad uruguaya, estimándose una venta de 26 millones de kilos al año. En 2005 los uruguayos gastaron en este rubro unos 46 millones de dólares. Al humedecerse, el residuo de yerba generado triplica su peso, por lo que se calcula que sólo en Montevideo se desechan diariamente unas 125 toneladas de yerba usada (Solari y Pelerin, 2006). Esto genera una gran cantidad de residuo orgánico con potencial de ser reciclado y que actualmente no es aprovechado, aumentando los problemas de acumulación de residuos vinculados con la higiene y la contaminación ambiental. En este escenario la lombricultura se presenta como una alternativa de reciclaje rápida y barata.
El empleo de abonos orgánicos para mejorar las cualidades físicas y químicas del suelo es una práctica antigua y de valor comprobado. El vermicompost es el producto final que resulta del proceso de descomposición de la materia orgánica realizado por algunas especies de lombrices. Es materia orgánica estabilizada o humificada rica en nutrientes y útil como abono natural y acondicionador de suelos. El humus de lombriz producido es un abono orgánico natural que mejora la porosidad y la retención de humedad en el suelo y aumenta la colonia bacteriana (Rodríguez, 1993). En su composición están presentes todos los nutrientes en cantidades suficientes para garantizar el desarrollo de las plantas. Favorece la circulación del agua y del aire en el suelo, facilitando la absorción de los elementos fertilizantes (Malvarez et al. 1994). A su vez, contiene sustancias fitorreguladoras que aumentan la capacidad inmunológica de las plantas. La actividad de las lombrices causa la transformación de la materia orgánica fresca a un estado de mineralización y esto puede ser medido por el incremento 
en la concentración de cenizas. La relación carbono/nitrógeno $(\mathrm{C} / \mathrm{N})$ aumenta con la presencia de lombrices, siendo 10 el valor que indica la relación óptima cuando el sustrato ha madurado en forma adecuada. Asimismo, la lombriz produce un aumento en el $\mathrm{pH}$ y en la capacidad de intercambio catiónico del sustrato (Schuldt, 2004. 151 p.)

El presente trabajo surge como propuesta del Centro Uruguay Independiente (CUI) en conjunto con el Laboratorio Tecnológico del Uruguay con el objetivo de aproximarse de manera preliminar a algunas de las condiciones necesarias para obtener compost de lombriz, a partir del residuo producido por el consumo de yerba mate, caracterizando tanto el sustrato inicial como el vermicompost final obtenido, desde el punto de vista físico-químico y microbiológico.

\section{Materiales y Métodos}

Para caracterizar el desecho yerba mate se instrumentó la recolección de este residuo generado por el personal del LATU durante todo un día (aproximadamente $600 \mathrm{~g}$ ). De esta manera se obtuvo un volumen de yerba fresca sobre la cual se analizaron los siguientes parámetros: $\mathrm{pH}$, humedad, nitrógeno, fósforo, potasio, y cenizas. Por otro lado también se obtuvieron desechos de yerba mate en un circuito de recolección previamente diseñado por el CUI, que abarcaba el barrio Cordón Norte de Montevideo. Dichos desechos (aproximadamente 50 kilos) se trasladaron al predio del LATU con una frecuencia semanal.

Se diseñaron tres etapas experimentales. La primera etapa consistió en la utilización de nueve tipos diferentes de sustratos, preparados a base de mezclas de yerba mate y estiércol en diferentes estados: yerba fresca recolectada en el día, yerba compostada que se dejó dejado reposar y airear 10 y 28 días respectivamente y, por último, estiércol compostado, estiércol vacuno que también se dejó reposar y airear durante 30 días. El estiércol fue suministrado por el tambo "Mundo al revés". Cada mezcla fue colocada en botellas plásticas de dos litros cortadas por la mitad. Las proporciones de las mezclas, el número de réplicas y la forma de identificación de los sustratos se muestran en la Tabla 1. Cada mezcla se inoculó con cinco lombrices juveniles (Eisenia fetida, de $5 \mathrm{~cm}$ de largo) que fueron pesadas en conjunto. Se registró el $\mathrm{pH}$ del sustrato y se cubrió el recipiente con gasas para evitar las posibles fugas, pero permitiendo una buena aireación. Los recipientes se acondicionaron en una cámara con temperatura y humedad constantes $\left(20^{\circ} \mathrm{C}\right.$ y $80 \%$ ) (Figura 1). A las 24 horas se suspendió el experimento al constatarse una mortandad de más del $90 \%$.

Como consecuencia de lo observado en la primera experiencia, se diseñó una segunda etapa utilizando otra serie de sustratos. En este caso, yerba que se mantuvo en reposo y aireada durante dos, tres y cuatro semanas respectivamente (yerba compostada), previo a su uso experimental. Se utilizaron baldes plásticos de cinco litros de capacidad, que se llenaron con $2 \mathrm{k}$ de sustrato y se inocularon con un total de 10 lombrices por balde, todas en estado adulto. Se registró el peso de cada grupo de 10 lombrices, así como el pH de cada tipo de sustrato. Se verificó que la humedad al comienzo de la experiencia fuera la adecuada, según el método de compresión de puño. Para cada sustrato se realizaron tres réplicas. Los recipientes experimentales se ubicaron en una cámara de temperatura y humedad controladas $\left(20^{\circ} \mathrm{C}\right.$ y $80 \%$ ). Cada 15 días se registró $\mathrm{pH}$ y peso de las lombrices presentes y se controló si la humedad del sustrato era la adecuada, regando con agua si fuese necesario. La experiencia se prolongó 90 días. Finalmente, para los análisis físico - químicos se realizó una mezcla de sustratos, utilizando las tres réplicas de cada tipo. Se procedió a analizar de dicha mezcla los siguientes parámetros: nitrógeno total, fósforo total, materia orgánica y la relación carbono/nitrógeno.

En una tercera etapa experimental y acorde a los resultados obtenidos en la segunda etapa, se utilizó yerba compostada por un período de 30 días previo al uso experimental. Se utilizaron dos cajones de madera de $0,87 \mathrm{~m}$ de largo, $0,70 \mathrm{~m}$ de ancho y $0,35 \mathrm{~m}$ de altura. En el cajón $\mathrm{N}^{\circ} 1$ se colocaron $68,3 \mathrm{~kg}$ de yerba inoculada con un conjunto de $0,47 \mathrm{~kg}$ de lombrices; en el cajón $\mathrm{N}^{\circ} 2$ se colocaron 72,7 kg de yerba y se inocularon con un conjunto de $0,47 \mathrm{~kg}$ de lombrices. Se tomaron registros semanales de $\mathrm{pH}$ durante 90 días. Se pesó también el total de lombrices que se obtuvieron por cajón. A ambas muestras de sustrato obtenidas se les realizaron los siguientes análisis: nitrógeno, fósforo, materia orgánica, relación carbono/nitrógeno, $\mathrm{pH}$, sulfatos, cromo, potasio, calcio, magnesio, cadmio, cobre, níquel, plomo, zinc y mercurio.

\begin{tabular}{|l|c|c|}
\hline \multicolumn{1}{|c|}{ Tipo de sustrato } & Toma (gr. & N de r $^{\circ}$ plicas \\
\hline yerba fresca & 200 & 6 \\
\hline yerba compostada (10d'as) & 150 & 6 \\
\hline yerba compostada (28 d'as) & 100 & 3 \\
\hline yerba fresca + 50\% de estiercol compostado & 150 & 6 \\
\hline yerba fresca + 25\% de bosta compostada & 150 & 6 \\
\hline yerba compostada (10d'as) + 50\% de estiercol compostado & 150 & 6 \\
\hline yerba compostada (10d'as) + 25\% de estiercol compostado & 150 & 6 \\
\hline yerba compostada (28 d'as) + 50\% de estiercol compostado & 150 & 3 \\
\hline yerba compostada (28 d'as) + 25\% de estiercol compostado & 100 & 3 \\
\hline
\end{tabular}

Tabla 1. Conformación de cada grupo experimental en la primera etapa experimental; se muestra el tipo de sustrato, las tomas realizadas, número de réplicas y $\mathrm{pH}$. Para la yerba fresca el $\mathrm{pH}$ es el que surge del informe de análisis químicos. El estiércol vacuno se obtuvo el 11 de octubre y se utilizó el 10 de noviembre.

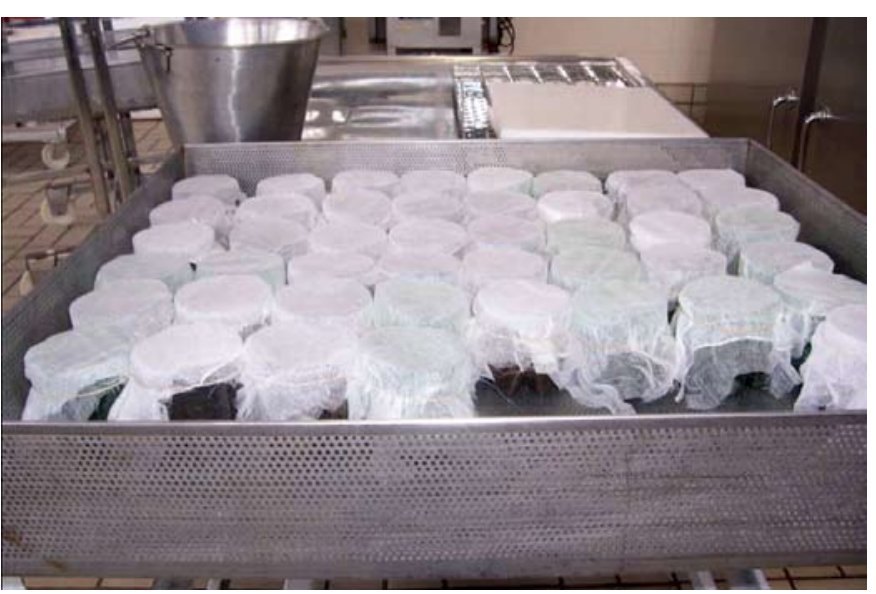

Figura 1. Bandeja conteniendo los recipientes de la primera etapa experimental listos para ser introducidos en la cámara de temperatura y humedad controladas.

\section{Resultados}

Los análisis de los residuos de yerba fresca recolectada en el LATU durante un día se muestran en la Tabla2, destacándose el bajovalor de $\mathrm{pH}$.

\begin{tabular}{|c|c|}
\hline Ensayo & Resultado \\
\hline $\mathrm{pH}$ & 4,2 \\
\hline Humedad $(\mathrm{g} / 100 \mathrm{~g})$ & 80,0 \\
\hline Fósforo (como $\mathrm{P})(\mathrm{mg} / \mathrm{Kg})$ & 260 \\
\hline Nitrógeno Kjeldahl $(\mathrm{como} \mathrm{N})(\mathrm{g} / 100 \mathrm{~g})$ & 4.2 \\
\hline Cenizas $(\mathrm{g} / 100 \mathrm{~g})$ & 1,6 \\
\hline Potasio $(\mathrm{g} / 100 \mathrm{~g})$ & 0,19 \\
\hline
\end{tabular}

Tabla 2. Análisis realizados sobre residuos de yerba mate frescos $(24 \mathrm{hs})$. 


\section{Primera etapa}

A las 24 horas de iniciada la experiencia se verificaron los recipientes y se comprobó la fuga de la inmensa mayoría de las lombrices de casi todos los recipientes. Se estimó conveniente suspender el experimento y comprobar cuántas lombrices vivas permanecían en cada mezcla. Se constató que solamente la muestra de yerba con $25 \%$ del estiércol, en dos pruebas compostadas 10 y 30 días, mantenían lombrices en cinco de las seis réplicas y en número variable. Se tomaron los valores de $\mathrm{pH}$, que se expresan en la Tabla 1. Considerando este primer resultado, y al no disponer de más estiércol vacuno, se diseñó la segunda etapa.

\section{Segunda etapa}

La variación de $\mathrm{pH}$ en los grupos con yerba compostada dos, tres y cuatro semanas se muestran en la Figura 2. Cabe aclarar que en el caso del grupo de dos semanas, las tres réplicas utilizadas mostraron el $100 \%$ de mortandad de lombrices en la primera semana del ensayo. Sin embargo, se continuó con las mediciones de $\mathrm{pH}$ en los recipientes sin lombrices para observar la variación en ausencia de lombrices. Los registros mostraron un incremento sostenido de los valores de $\mathrm{pH}$ (grupo dos semanas y grupo tres semanas), tornándose más básico hasta el día 20, estabilizándose y descendiendo levemente hasta el valor aproximado de 7,5 próximo a los 100 días de experiencia. El grupo correspondiente a las cuatro semanas parte de un valor de $\mathrm{pH}$ próximo a 8 y continúa subiendo hasta el día 30 , a partir del cual se ve un descenso pronunciado hasta el día 60 , cuando se estabiliza hasta el valor final de 7,5 aproximadamente.

\section{Variación de $\mathrm{pH}$ promedio por grupo experimental}

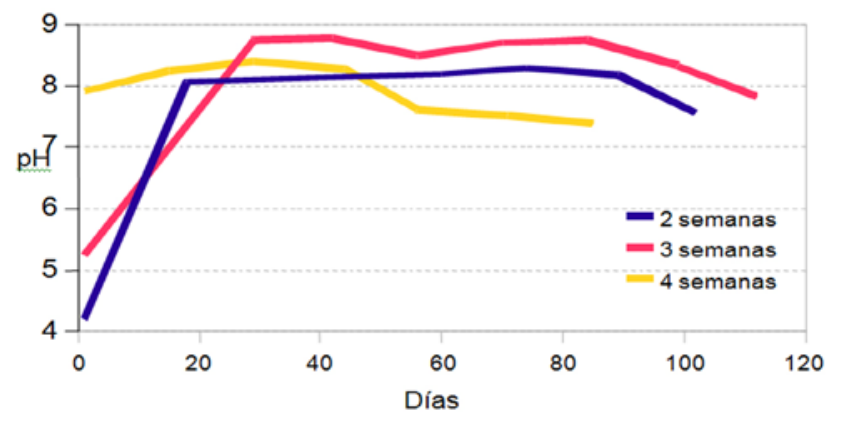

Figura 2. Variación del pH promedio de cada grupo en función del tiempo, días correspondientes a los grupos experimentales de dos, tres y cuatro semanas.

Con respecto a la variación en la biomasa, el grupo correspondiente a yerba compostada tres semanas presentó una reducción importante del número de lombrices en la primera semana de registros $\mathrm{y}$ posteriormente una mortandad del $100 \%$ en dos de las tres réplicas (Figura 3).

El grupo yerba compostada cuatro semanas mantuvo la población de lombrices estable hasta los 60 días aproximadamente; a partir de ese momento se observó un incremento considerable de la población de juveniles, lo cual se vio reflejado en un aumento sostenido en la biomasa total (Figura 4). Se observó presencia de cocones en todas las réplicas de este grupo a partir del mes y medio de comenzada la experiencia.

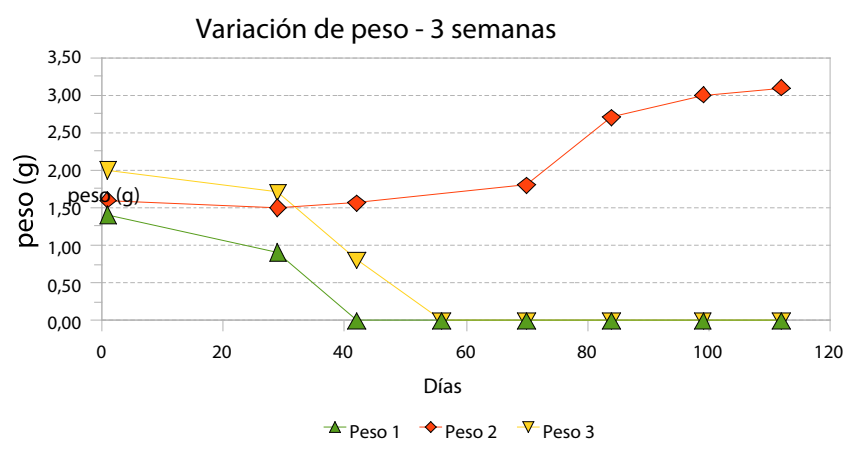

Figura 3. Variación de biomasa de las lombrices correspondiente al grupo yerba compostada de tres semanas. Dos de las réplicas alcangrupo yerba compostada de tres semanas. Dos de las replicas alcanexperiencia. Peso 1 , peso 2 y peso 3 corresponden a las distintas réplicas.

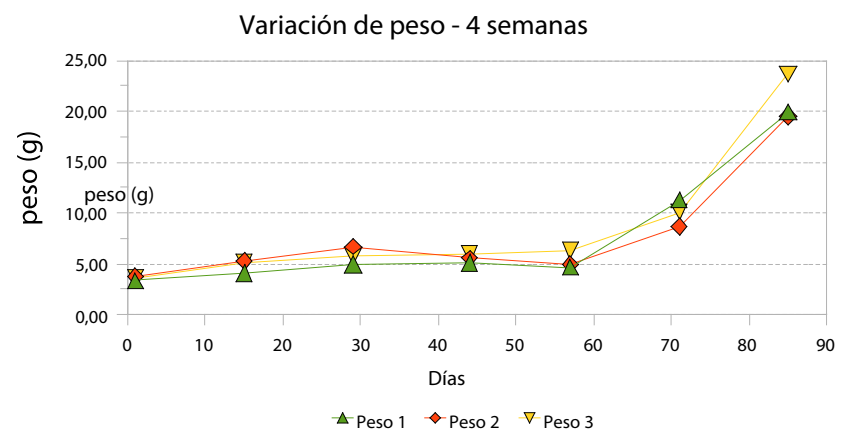

Figura 4. Variación de biomasa correspondiente al grupo yerba compostada de cuatro semanas. Peso 1, peso 2 y peso 3 corresponden a las distintas réplicas

Al finalizar la experiencia se analizaron químicamente los pools de réplicas de los sustratos correspondientes a las muestras de los dos grupos de yerba compostada de tres y cuatro semanas respectivamente, ya que fueron las únicas que mantuvieron poblaciones de lombrices vivas. Los resultados muestran similitudes en los valores de la mayoría de los parámetros medidos, excepto por una pequeña diferencia en el fósforo total (Tabla 3). De acuerdo a los resultados obtenidos en estos ensayos, se diseñó la tercera etapa experimental.

\begin{tabular}{|c|c|c|}
\hline Ensayo & Semana 3 & Semana 4 \\
\hline $\begin{array}{c}\text { Materia orgánica } \\
\text { base seca }(\mathrm{g} / 100 \mathrm{~g})\end{array}$ & 27,6 & 23,7 \\
\hline Fósforo (como P) (mg/kg) & 633 & 526 \\
\hline $\begin{array}{c}\text { Nitrógeno Kjeldahl (como N) } \\
(\mathrm{g} / 100 \mathrm{~g})\end{array}$ & 1,3 & 1,2 \\
\hline Relación C/N & 12,3 & 12,4 \\
\hline
\end{tabular}

Tabla 3. Resultados de los ensayos realizados al pool de compost obtenido en la segunda etapa.

\section{Tercera etapa}

Los registros del $\mathrm{pH}$ de los cajones donde se realizaron los ensayos con yerba de 30 días se muestran en la Figura 5. El comportamiento 
inicial fue un descenso en el valor del $\mathrm{pH}$ durante los primeros 35 días, hasta un valor aproximado de 7,2. Posteriormente aumentó el valor y se estabilizó cercano a 8 .

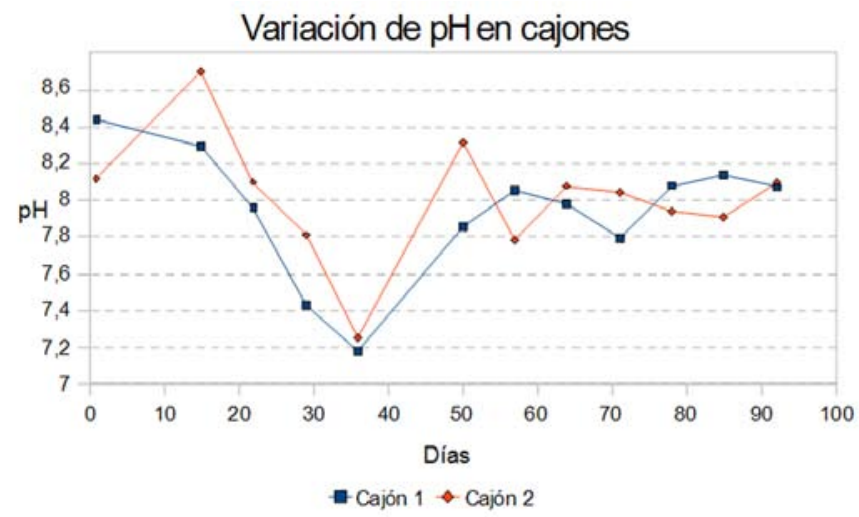

Figura 5. Variación del pH en función del tiempo, correspondiente a la tercera etapa realizada en cajones.

Una vez terminado el experimento se analizaron los parámetros físico-químicos del vermicompost resultante, los cuales se detallan en la Tabla 4.

\begin{tabular}{|c|c|c|}
\hline Ensayo & Cajón 1 & Cajón 2 \\
\hline Materia orgánica base seca $(\mathrm{g} / 100 \mathrm{~g})$ & 30,2 & 25,5 \\
\hline Fósforo (como P) (mg/kg) & 439 & 452 \\
\hline Nitrógeno Kjeldahl (como N) (g/100g) & 1,3 & 1,1 \\
\hline Relación $\mathrm{C} / \mathrm{N}$ & 13,5 & 13,4 \\
\hline $\mathrm{pH}$ & 7,96 & 7,36 \\
\hline Sulfatos $($ como SO4)(mg/kg) & 2,70 & 2,82 \\
\hline Cromo (mg/kg) & $<5,0$ & $<5,0$ \\
\hline Potasio (mg/kg) & $9,5 \mathrm{E}+03$ & $7,7 \mathrm{E}+03$ \\
\hline Calcio (mg/kg) & $1,5 \mathrm{E}+04$ & $1,7 \mathrm{E}+04$ \\
\hline Magnesio (mg/kg) & $8,8 \mathrm{E}+03$ & $5,4 \mathrm{E}+03$ \\
\hline Cadmio (mg/kg) & $<2,0$ & $<2,0$ \\
\hline Cobre $(\mathrm{mg} / \mathrm{kg})$ & $<60$ & $<60$ \\
\hline Níquel (mg/kg) & $<4,0$ & $<4,0$ \\
\hline Plomo (mg/kg) & $<5,0$ & $<5,0$ \\
\hline Zinc $(\mathrm{mg} / \mathrm{kg})$ & $<2,0$ & $<2,0$ \\
\hline Mercurio (mg/kg) & $<1,8$ & $<1,8$ \\
\hline
\end{tabular}

Tabla 4. Ensayos realizados al vermicompost obtenido en la tercera etapa experimental

\section{Discusión y Conclusiones}

El presente proyecto buscó determinar de manera muy básica aquellas condiciones más favorables para la producción de compost a partir de residuos de yerba mate como sustrato y a la lombriz de tierra roja (Eisenia fetida). Los procedimientos aquí utilizados pretendieron contemplar a los empleados por el CUI en cuanto a las etapas piloto se refiere (Solari y Pelerin, 2006). Por último se analizaron las características físico-químicas del humus resultante.

La primera etapa experimental planteó problemas en la adaptación de la lombriz a los diferentes sustratos propuestos. Se evidenció una gran mortandad a las 24 horas de comenzada la experiencia, observándose un abandono de los recipientes de forma masiva. Los sustratos estaban compuestos por diferentes porcentajes de yerba y estiércol vacuno, con $\mathrm{pH}$ iniciales próximos a 8 en la mayoría de los casos. Las mezclas que presentaron valores de $\mathrm{pH}$ más ácidos fueron las que tenían yerba fresca y estiércol compostado. Los sustratos fueron elaborados a partir de mezclas que se hicieron el día del inicio de las experiencias, encontrándose la dificultad en la homogeneización de la yerba con el estiércol, y posiblemente la medición de $\mathrm{pH}$ no fue muy precisa. No se descarta que otros factores tal como el tamaño reducido de los recipientes y la falta de escurrimiento pudieran causar anoxia y posterior mortandad, o bien una gran variación posterior del $\mathrm{pH}$ en el sustrato debida a una mala homogeneidad, causaron la fuga y muerte de la mayoría de las lombrices. El rediseño experimental consideró utilizar recipientes de mayor tamaño y no usar estiércol, ya que no se disponía del mismo en el tiempo necesario.

La segunda etapa mostró una muerte masiva en el grupo correspondiente a la yerba compostada durante dos semanas. Se conoce que el rango de $\mathrm{pH}$ tolerable de Eisenia fetida se encuentra en el entorno de 5 y 8,5 , encontrándose un $\mathrm{pH} 4$ al inicio del ensayo, lo cual pudo afectar la sobrevivencia de este grupo.

Durante la etapa experimental en cajones el comportamiento del $\mathrm{pH}$ fue parecido en ambas réplicas y como era esperable en un proceso de compostaje. Las curvas de $\mathrm{pH}$ a lo largo del tiempo coincidieron en estabilizarse en valores entre 7 y 8 al final del tercer mes de experiencia. Esta característica química del sustrato coincidió con la aparición de posturas (cocones) y de los primeros juveniles.

En base a estas primeras observaciones experimentales, parecería conveniente que previo a la introducción de lombrices, se dejara reposar la yerba fresca durante por lo menos un mes, manteniendo la humedad de la misma, hasta lograr la estabilización del pH. De esta forma sería posible una mejor adaptación de las lombrices al sustrato dentro de un rango tolerable de $\mathrm{pH}$, disminuyendo la mortandad y permitiendo el desarrollo de los estadios juveniles. Dado al diseño experimental planteado, el cual intenta reducir la manipulación directa de las lombrices, no fue posible estimar los efectos causados por tal perturbación. Por lo tanto este puede ser un factor más a tener en cuenta dentro de las variables que afectan los resultados obtenidos.

La calidad del humus bajo estos tratamientos experimentales se comparó con resultados analíticos de otros trabajos y de ensayos previos del vermicompost obtenido por el CUI, según su método de trabajo. El valor de nitrógeno (1\%) y de materia orgánica, que ronda el $30 \%$, es similar al encontrado en otros humus que se obtienen por procedimientos semejantes. En cambio, para el fósforo los valores medidos en el presente trabajo resultaron por debajo de lo encontrado en el análisis realizado por CUI (Laboratorio Ambiental Echotech, octubre 2006).

En Uruguay no se encontraron normas que establezcan las características que debe tener el humus obtenido a partir de lombrices para ser comercializado. En general, cada país tiene sus propios límites establecidos dentro de los parámetros de caracterización, dependiendo del uso que tenga el humus obtenido, ya sea para cultivos, viveros, etcétera. Si bien es difícil encontrar estándares sobre humus de lombriz, se puede comparar con estándares de humus en general. La variación de la calidad del humus de lombriz está dada por varios factores, uno de los más importantes es la dieta (sustrato), donde se realiza la transformación. En este trabajo los niveles de metales encontrados se encuentran por debajo de los rangos permitidos para compost dentro de la Unión Europea y para Estados Unidos (Briton, 2000).

Cabe aclarar que este es un estudio preliminar para conocer algunas de variables que afectan la producción de vermicompost a partir de residuos de yerba mate. Sin embargo y en base a lo observado, la cantidad de materia orgánica obtenida por este proceso de vermicultura parece ser baja comparada con los demás humus obtenidos por procesos de compostaje. De todas formas, si se considera la facilidad en el manejo para obtener este producto y el bajo costo en la inversión 
inicial, parece ser un sistema adecuado para su aplicación a nivel doméstico y de pequeñas huertas o viveros, redundando en beneficios a nivel de aporte de nutrientes al suelo y aprovechando un residuo tan frecuente y abundante en los hogares uruguayos como la yerba mate.

\section{Reconocimientos}

Agradecemos a la editora Marina Barrientos y a los revisores anónimos por la lectura crítica del manuscrito. A Flavio Pelerino por sus sugerencias y contribuciones al proyecto conjunto con la CUI. Por último, agradecemos a todo el personal del LATU que de una u otra manera hicieron posible llevar adelante este trabajo.

\section{Referencias}

- BRITON, W. Compost quality standards and guidelines: final report [En línea]. Mount Vernon: Woods End Research Laboratory, 2000. [Consulta: 3 de octubre de 2008]. Disponible en: http://compost.css.cornell.edu/Brinton.pdf

- FOGAR, M. N.; CRACOGNA, M. F.; IGLESIAS, Mariano. Respuesta de la lombriz roja (Eisenia foetida) frente a diferentes alimentos. Corrientes: Facultad de Ciencias Agrarias-UNNE, 2000.

- HERNÁNDEZ, José Danilo; MONTES DE OCA, Paulina; VILLALOBOS, Cecilia. Estudio reproductivo de la lombriz de tierra Eisenia foetida en cuatro sustratos, en la finca experimental Santa Lucía, Universidad Nacional. En: Colegio de Ingenieros Agrónomos de Costa Rica. X Congreso Agronómico Nacional y de Recursos Naturales, (San José 8-12 de julio de 1996). San José: Colegio de Ingenieros Agrónomos de Costa Rica, 1996.

- MALVAREZ, G.; ZORRILLA, A.; RODRIGUEZ, A. Alternativas de abonos orgánicos: compostaje con lombrices. Montevideo: Escuela Agroecológica Programa Norte, 1994.

- MUNROE, Glenn. Manual of on-farm vermicomposting and vermiculture [En línea]. Truro: Organic Agriculture Centre of Canada, 2004. [Consulta: 3 de octubre de 2008]. Disponible en: http://oacc.info/DOCs/Vermiculture_FarmersManual_gm.pdf

- RECALDE, Luis A. Proyecto: lombrices californianas (Eisenia foetida) [En línea]. [s.l.]: [s. n], [s.d]. [Consulta: 3 de octubre de 2008]. Disponible en: http://www.monografias.com/trabajos10/ lombri/lombri.shtml?relacionados

- RODRÍGUEZ, Angel Ramón. Producción y calidad de abono orgánico por medio de la lombriz roja californiana (Eisenia Foetida) y su capacidad reproductiva [En línea]. [Consulta: 3 de octubre de 2008]. Disponible en: www.fao-sict.un.hn/practicas/unload/944.pdf

- SCHULDT, M., et al. Pruebas de aceptación de alimentos y constraste de dietas en lombricultura. En: RedVet. 2005, 6(7):1-12.

- SOLARI, Jorge; PELERINO, Flavio. La yerba no es basura: lombrices, humus y cultivos urbanos. Montevideo: Centro Uruguay Independiente, 2006.

- TOCCALINO, Pedro A.; ROUX, Juan P.; AGÜERO, Celeste M. Comportamiento reproductivo de Eisenia foetida durante las cuatro estaciones del año y alimentadas con distintos compostajes [En línea]. Corrientes: Facultad de Ciencias Veterinarias, 1999. [Consulta: 3 de Octubre de 2008]. Disponible en: http:/www.bio-nica.info/Biblioteca/Toccalino1999.pdf 